\title{
Questioning the acute effect of orange chromatic environment on perceived health status, pain, and vital signs during chemotherapy treatment
}

\author{
Jun Kako ${ }^{1}$ (D) Kohei Kajiwara ${ }^{1} \cdot$ Masamitsu Kobayashi $^{2} \cdot$ Yasufumi Oosono $^{3}$ \\ Received: 4 September 2019 / Accepted: 16 September 2019 / Published online: 16 October 2019 \\ (C) Springer-Verlag GmbH Germany, part of Springer Nature 2019
}

To the Editor:

We read the recent study by Gómez-Vela et al. [1] on the acute effect of orange color and natural light exposure on cancer patients during chemotherapy sessions with great interest. The authors concluded that compared with a cool-color design, a warm-color living environment could have a positive effect on patients' well-being during chemotherapy sessions. We would like to raise a few questions that possibly affected the results.

First, the control group setting was questionable. The study was designed to compare between two warm rooms (orangenorth and orange-south) and two cool rooms (white-north and white-south). However, we believe that it is necessary to compare warm rooms with the environment where usual chemotherapy is received.

Second, information about the weather was not described. The authors installed 3M translucent vinyl sheets on the glasses, but we are concerned that the amount of light may change depending on the weather.

Third, information about the participant's color preference was not described. Some people like warm colors and others like cool colors, and this may affect the results.

Jun Kako

jkako@hiroshima-u.ac.jp

1 Division of Nursing Science, Graduate School of Biomedical and Health Sciences, Hiroshima University, 1-2-3, Kasumi, Minami-ku, Hiroshima 734-8553, Japan

2 Faculty of Nursing, National Defense Medical College, Tokorozawa, Japan

3 Community Health Nursing, National Defense Medical College, Tokorozawa, Japan
The possibility that side effects from chemotherapy can be relieved by environmental adjustment, such as color changes, is really interesting. We believe that additional discussion of these questions could clarify the results in the study by Gómez-Vela and colleagues.

\section{Compliance with ethical standards}

Conflict of interest The authors declare that they have no conflict of interest.

\section{Reference}

1. Gómez-Vela P, Pérez-Ruiz M, Hernández Martín MF, Román J, Larumbe-Zabala E (2019) Acute effect of orange chromatic environment on perceived health status, pain, and vital signs during chemotherapy treatment. Supportive Care in Cancer:1-9. https://doi.org/10. 1007/s00520-019-05064-w

Publisher's note Springer Nature remains neutral with regard to jurisdictional claims in published maps and institutional affiliations. 